\title{
Laryngeal Tuberculosis Revealed by Laryngeal Dyspnea
}

\author{
Belhaj $\mathrm{N}^{1,3^{*}}$, Lassikri $\mathrm{O}^{1}$, Nekro $\mathrm{C}^{1}$, Benkhrab $\mathrm{N}^{1}$, Bencheikh $\mathrm{R}^{2}$, Benbouzid $\mathrm{MA}^{2}$, Essakalli $\mathrm{L}^{2}$
}

${ }^{1}$ Resident physician in otorhinolaryngology, Department of Otorhinolaryngology, Head and Neck Surgery, Ibn Sina University Hospital, Rabat, Morocco

${ }^{2}$ Professor of otorhinolaryngology, Department of Otorhinolaryngology, Head and Neck Surgery, Ibn Sina University Hospital, Rabat, Morocco

${ }^{3}$ Faculty of Medicine and Pharmacy of Rabat, Mohammed V University, Rabat, Morocco

DOI: $10.36347 /$ sjmcr.2020.v08i03.036

| Received: 12.03.2020 | Accepted: 20.03.2020 | Published: 24.03.2020

*Corresponding author: Belhaj $\mathrm{N}$

Abstract

Isolated laryngeal tuberculosis is rare, it can be considered as a malignant tumor which delay the diagnosis and worsens the prognosis. We report the case of a 31-years-old patient admitted to the emergency room for the management of a progressive laryngeal dyspnea, with a laryngoscopic examination confirmed an erythematous and thick appearance of the two vocal cords. The histological examination revealed epitheloid and giganto-cellular granulomas with a caseous necrosis suggestive of tuberculosis. Culture on lowenstein medium of tissue biopsied from the vocal cords was positive. No other tuberculosis localization was found. The patient underwent standard antituberculosis therapy (isoniazid, rifampicin, ethambutol, pyrazinamide), which completely resolved the clinical situation.

Keywords: Laryngeal Tuberculosis Laryngeal Dyspnea.

Copyright @ 2020: This is an open-access article distributed under the terms of the Creative Commons Attribution license which permits unrestricted use, distribution, and reproduction in any medium for non-commercial use (NonCommercial, or CC-BY-NC) provided the original author and source are credited.

\section{INTRODUCTION}

Tuberculosis (TB) is a contagious infectious disease of chronic evolution caused by Mycobacterium tuberculosis. Tuberculosis affects mainly the lungs but can occur in any organ. Tuberculosis is only rarely confined to the larynx in the absence of associated lung damage. Clinicians should be familiar with this unusual site given the global upsurge of tuberculosis in the past 20 years, mainly facilitated by the spread of infection with the human immunodeficiency virus (HIV) and also due to the diagnostic difficulty caused by this unusual site of tuberculosis, which is rarely considered and which can be confused with laryngeal cancer. Clinical and endoscopic examinations are not specific in the case of laryngeal tuberculosis. Only the typical histological results or the bacteriological results make it possible to confirm the tuberculous origin of the laryngeal symptomatology. The objectif of this study is to emphasize the difficulty of the diagnosis of laryngeal tuberculosis which can simulate neoplasia, vasculitis, sarcoidosis, lymphoma or another infection.

\section{Description OF THE CASE}

We report in this work the case of a 31-yearsold patient who was admitted to the emergency room for respiratory distress with laryngeal stridor. The first symptoms to appear were dysphonia since 1 year (gradually getting worse), without dysphagia, and progressive dyspnea complicated of respiratory distress. The patient had a tracheotomy, and then she benefited from a panendoscopic examination which objectified a cystic mass in the retro-crico-arytenoid region invading the vocal cords and the ventricular folds with subglottic involvement. Histological examination (of the biopsies made from the vocal cords) found inflammatory infiltrates organized in the form of granulomas associating lymphocytes, epitheloid cells and giant cells, fibrosis and necrosis caseous. No signs of malignancy or vasculitis were identified. These results were suggestive of the diagnosis of laryngeal tuberculosis. Ziehl Neelsen's staining revealed acid-fast bacilli (AFB). The culture of injured laryngeal tissues on Lownstein medium was positive. No other tuberculosis localization was found either by physical examination or by imagery. The cervico-thoracic CT scan(Fig1) showed a thickening of the left vocal cord reducing the glottic light, the cervical MRI (Fig2)showed a circumferential stenosing glotticsubglottic laryngeal process interesting the vocal cords and the ventricular folds predominant on the left extended at the anterior and posterior commissure with bilateral jugulo carotid and supraclavicular adenoapthias. 


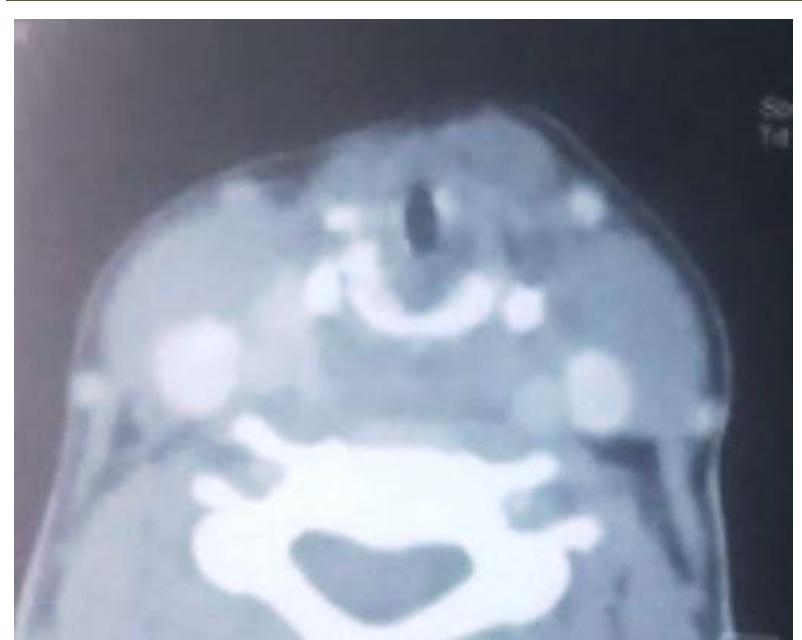

Fig-1: CT scann axial section showing tissue thickening of the left vocal cord extended below the glottis

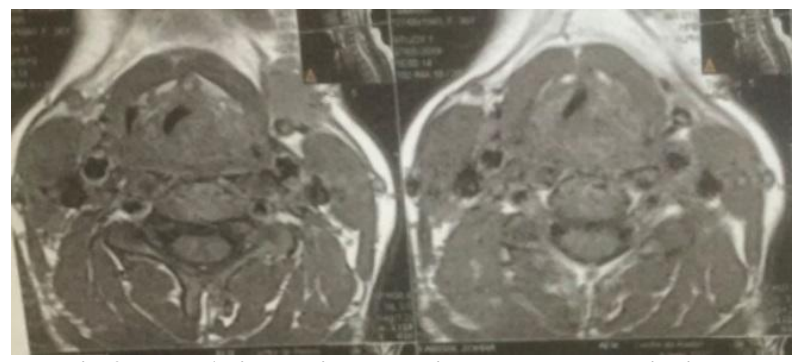

Fig-2: MRI Axial sections showing glotto subglottic tissue thickening

\section{DISCUSSION}

Tuberculosis is a Mycobacterium tuberculosis infection whose localization is mainly pulmonary. It can occur in immunocompetent patients, or most often immunocompromised. Tuberculosis can manifest itself in a latent and chronic form leading to irreducible tissue damage or even miliary tuberculosis in case the diagnosis is delayed. Vaccination against tuberculosis has reduced the incidence of this disease. However, some areas are still considered endemic areas where the diagnosis of tuberculosis should be considered [1]. Thus, the vaccine profile should be checked especially in immunocompromised patients who live in these areas. Therefore, the clinical characteristics of tuberculosis have been changed due to the availability of anti-tuberculosis drugs and the increased frequency of immunosuppressive conditions. These conditions have favored the emergence of forms of extrapulmonary tuberculosis involving the spine, muscles, meninges, pituitary gland, thyroid, digestive system, hematopoietic organs, larynx, sinuses, nose $[1,2]$.

Isolated laryngeal tuberculosis sites are rare [3]. In fact, laryngeal tuberculosis is always associated with pulmonary involvement and generally occurs in patients without BCG vaccination or in case of an immune deficiency [3-5]. Primary laryngeal tuberculosis can manifest as chronic hoarseness or dysphonia in a febrile context. El Ayoubi F et al. studied ten cases of primary laryngeal tuberculosis. In this series, the patients were male smokers. They had no specific clinical or endoscopic signs. The clinical pictures of these patients simulated those of larynx cancer. Only bacteriological and histological examinations allow diagnostic confirmation [4]. Malignant neoplasm, lymphoma or vasculitis such as Wegener's disease and sarcoidosis are considered the main differential diagnoses of laryngeal tuberculosis. Endoscopic results may show ulcerations or vegetations. The series of studies by Hasibi $\mathrm{M}$ et al. revealed that the macroscopic aspects of laryngeal lesions were vegetative in 11 cases and ulcerative in 14 cases [5]. Symptoms and clinical signs are not specific with an insidious beginning suggestive of simple laryngitis. The dysphonia is rapidly progressive, and sometimes it evolves rapidly towards aphonia, such is the case of our patient who presented at the beginning a progressive worsening dysphonia progressing to dyspnea. Dyspnea is now an extremely rare symptom, reflecting advanced and neglected disease. Odynophagia is reported in $25 \%$ to $50 \%$ of cases in the literature, even in the early stages of the disease. The severity of odynophagia was correlated according to the degree of involvement of the aryepiglottic folds and hypopharynx. Odynophagia worsens gradually, starting with painful swallowing of saliva, associated with a fungal, ulcerative lesion, or even perichondritis of the epiglottis, cricoid or arytenoid. Note that the combination of odynophagia and progressive dysphonia strongly suggests the diagnosis. The cough is variable depending on the lesions. Typically, the patient experiences a dry laryngeal cough with a feeling of foreign body associated with hemoptysis. The cough was not reported by the patient [6].

Systemic signs are often minor in the primary forms of laryngeal tuberculosis and deterioration of the general state (fever, asthenia, weight loss) is mainly observed in advanced forms with odynophagia. The examination of the larynx is essentially based on the direct laryngoscopy with biopsy to clarify the site and appearance of tuberculous lesions. The biopsy confirms the histological diagnosis and excludes cancer, based on the appearance of the lesions, the age of the patient and a history of alcohol and tobacco abuse. Recent publications show that laryngeal tuberculosis involves the vocal cord ligament then, in decreasing order of frequency, the vestibular fold and the epiglottis, in the form of epiglottic tuberculosis. The epiglottis is then infiltrated and swollen causing severe odynophagia. The subglottis and the posterior commissure may also be involved. In the past, laryngeal tuberculosis mainly involved the posterior part of the larynx, more precisely the posterior commissure [6]. Laryngeal lesions have a non specific appearance, which explains the problems of differential diagnosis with larynx cancer, especially in early, ulcerative and fungal forms [7]. Edema and hyperemia are observed in the early stages of the disease. More advanced forms were classically associated with an appearance of "coated larynx", with 
a greyish and purulent coating, with a large lesion occupying most of the larynx includes infiltrated areas and ulcerated areas. Direct laryngoscopy remains the key to diagnosis it allows to perform biopsies and send them for bacteriological and histological study [8]. Ziehl Neelson's staining showing acid-fast bacilli and positive specific culture on Lownstein medium are good arguments for retaining the tuberculous origin. The histological results can be typical, as presented in our case (chronic granulomatous inflammation with giant cells, presence of caseous necrosis and Langhans type cells) confirming the diagnosis or atypical (chronic granulomatosis inflammation or chronic inflammation without necrosis) responsible for diagnostic delay [9, 10]. Radiological examinations contribute little to the positive diagnosis and $\mathrm{CT}$ with improved contrast remains the reference, it allows a precise topographic evaluation and staging of lesions in the glottic region and laryngeal cartilages, as well as the evaluation of any complications in particular glottic stenosis or subglottic. A general assessment should be made in order to eliminate a secondary form of laryngeal tuberculosis. This assessment consists of looking for a personal and family history, doing a chest x-ray and looking for BK in the sputum and in the gastric fluid 3 days followed $[11,12]$. An examination of the cervical ganglionic areas in search of lymphadenopathy, especially in the areas under digastrics, which are the drainage site of the upper aerodigestive tract.

Many differential diagnoses can be made, especially cancer of the larynx, more often proposed in the presence of an ulcerative, fungal, papillomatous or pseudoneoplastic lesion, especially in patients with a history of alcohol and tobacco abuse. A doubt may even persist after histological examination of biopsies when they only comprise superficial layers. Biopsies should be repeated when the histological examination is inconclusive. Physicians should also keep in mind that carcinoma and tuberculosis can coexist in the same patient. Several cases of concurrent carcinoma and tuberculosis have been reported in the literature.

Tuberculosis can be confused with infectious laryngitis. Other diagnoses are exceptional to consider in the presence of granulomatous lesions of the larynx are syphilis, amyloidosis, actinomycosis, blastomycosis of the larynx and Wegener's disease [8, 13].

After confirming the positive diagnosis of laryngeal tuberculosis, treatment should be started as soon as possible with anti-tuberculosis chemotherapy for six months generally consisting of: isoniazid $(5 \mathrm{mg} /$ $\mathrm{kg}$ ), rifampicin $(10 \mathrm{mg} / \mathrm{kg}$ ) and pyrazinamide (20 at 30 $\mathrm{mg} / \mathrm{kg}$ ) for the first two months and dual therapy including isoniazid and rifampicin at the same doses for the following four months (8). Corticosteroids can also be prescribed in certain situations such as the presence of lymphadenopathy. Some measures must be taken to ensure adherence to treatment, such as the use of the
DOT approach or direct observation therapy (DOT), as in some countries where tuberculosis is endemic. In this concept, the patient visits a health center near his home every day for the first time for two months to receive treatment on an empty stomach, in the presence of a witness specially trained for this role. The initiation of TB treatment should be preceded by a detailed pretreatment assessment and should include surveillance for treatment complications. In particular, surveillance of hepatic and renal function, these functional tests must be carried out after two weeks, 2 months and 6 months; an ophthalmological examination must be carried out after 2 months, bacteriological examinations must be carried out after 2, 4 and 9 months, endoscopic monitoring of lesions of the larynx and serum isoniazid dosage must be carried out in patients who have renal or hepatic insufficiency, or neuropsychiatric disorders. Surgery is no longer part of the current treatment options (14), with the exception of tracheostomy in patients with very severe dyspnea or to treat the sequelae after medical treatment, as fibrotic scarring can leave scars that can be responsible for arytenoid immobility when they interfere with a cricoarytenoid joint, or glottic or subglottic stenosis, especially in the presence of cricoid scars. Once the diagnosis is confirmed, anti-tuberculosis treatment administered for a minimum of 6 months has given excellent short-term and long-term results. This duration was extended in our case to twelve months to avoid irreducible tissue destruction of the larynx [14].

\section{CONCLUSION}

It's important to consider laryngeal tuberculosis as a rare infectious disease with variable clinical signs and polymorphic endoscopic results, but for which there is very effective medical treatment.

\section{REFERENCES}

1. Feleke Y, Abdulkadir J, Aderaye G. Prevalence and clinical features of tuberculosis in Ethiopian diabetic patients. East Afr Med J. 1999; 76(7):3614

2. Podell BK, Ackart DF, Obregon-Henao A, Eck SP, Henao-Tamayo M, Richardson M, Orme IM, Ordway DJ, Basaraba RJ. Increased severity of tuberculosis in Guinea pigs with type 2 diabetes: a model of diabetes-tuberculosis comorbidity. The American journal of pathology. 2014 Apr 1;184(4):1104-18.

3. Mahfoudhi M, Khamassi K, Turki S, Kheder A. Difficulté diagnostique d'une tuberculose laryngée isolée chez une femme diabétique. Pan African Medical Journal. 2015;21(1).

4. El Ayoubi F, Chariba I, El Ayoubi A, Chariba S, Essakalli L. Primary tuberculosis of the larynx. Eur Ann Otorhinolaryngol Head Neck Dis. 2014; 131(6): 361-364. 
5. Hasibi M, Yazdani N, Asadollahi M, Sharafi M, Manshadi SA. Clinical features of laryngeal tuberculosis in Iran. Acta Medica Iranica. 2013:638-41.

6. Gallas D, Coste A, Bedbeder P, Peynegre R. Aspects actuels de la tuberculose laryngée: à propos de quatre cas et revue de la littérature. InAnnales d'oto-laryngologie et de chirurgie cervico-faciale 1994 (Vol. 111, No. 4, pp. 201207).

7. Hunter AM, Millar JW, Wightman AJ, Horne NW. The changing pattern of laryngeal tuberculosis. The Journal of Laryngology \& Otology. 1981 Apr;95(4):393-8.

8. Tattevin P, Crémieux AC, Bouvet E, Carbon C. La tuberculose: actualités thérapeutiques. La Presse médicale (1983). 1996;25(39):1909-15.

9. Schluger NW. Changing approaches to the diagnosis of tuberculosis. American journal of respiratory and critical care medicine. $2001 \mathrm{Dec}$ 1;164(11):2020-4.

10. Leroux-Robert J, de Brux J. Histopathologie ORL et cervico-faciale. Masson; 1976.

11. Moon WK, Han MH, Chang KH, Im JG, Kim HJ, Sung KJ, Lee HK. CT and MR imaging of head and neck tuberculosis. Radiographics. 1997 Mar;17(2):391-402.

12. El Kettani NE, El Hassani M, Chakir N. Primary laryngeal tuberculosis mimicking laryngeal carcinoma: CT scan features. Indian J Radiol Imaging. 2010;20(1):11-2

13. Lin CJ, Kang BH, Wang HW. Laryngeal tuberculosis masquerading as carcinoma. European archives of oto-rhino-laryngology. 2002 Nov 1;259(10):521-3.

14. Herzog JA, Donovan DT. Complete subglottic stenosis secondary to tuberculosis. Otolaryngol Head Neck Surg. 1985;93:666-9. 